April 2007

\title{
Electroweak Higgs as a pseudo-Goldstone boson of broken scale invariance
}

\author{
Robert Foot, Archil Kobakhidze and Raymond R. Volkas ${ }^{1}$ \\ School of Physics, Research Centre for High Energy Physics, \\ The University of Melbourne, Victoria 3010, Australia
}

\begin{abstract}
We point out that it is possible to associate the electroweak Higgs boson with the pseudoGoldstone boson of broken scale invariance, thus resolving the hierarchy problem in a technically natural way. We illustrate this idea with two specific gauge models. Besides being consistent with all currently available experimental data, both models maintain the predictive power of the standard model, since the first model has only one additional parameter beyond the standard model, and the second has the same number of free parameters as the standard model.
\end{abstract}

\footnotetext{
${ }^{1}$ E-mail: foot@physics.unimelb.edu.au, archilk@physics.unimelb.edu.au, r.volkas@physics.unimelb.edu.au
} 


\section{Introduction}

Understanding the origin of mass is one of the key problems within the standard model (SM). The chiral nature of the gauge symmetry of the Standard Model forbids masses for quarks and leptons, apart from right-handed neutrino Majorana masses. Taking neutrinos to be Dirac, the only mass parameter in the theory is the electroweak $\mu^{2}$ mass parameter in the Higgs potential. Setting it to zero renders the SM scale invariant at the classical level. However, the scale invariance is known to be anomalous; it is broken at the quantum level. This manifests through the important effect called dimensional transmutation. In particular, in a scale invariant theory a scalar field can develop a vacuum expectation value (VEV) radiatively as a result of the quantum conformal anomaly [1]. On general grounds one can argue that the existence of a light scalar field, with its mass generated entirely at loop level, is inevitable within scale invariant models that involve also multiple scalar fields [2]. This light scalar field is nothing but a pseudo-Goldstone boson (PGB) accompanying the breaking of the anomalous scale invariance. It is tempting to associate this PGB with the electroweak Higgs boson. The reasons are obvious. Firstly, a massless theory is more predictive than the corresponding theory with a priori unknown mass parameters. Secondly, and perhaps more importantly, the electroweak scale generated through the dimensional transmutation will be radiatively stable, thus resolving the technical aspect of the hierarchy problem. Unfortunately, this very appealing theoretical framework applied within the SM predicts a very light Higgs boson with $m_{\mathrm{h}}<10 \mathrm{GeV}$ and also requires the top quark to be light: $m_{\mathrm{t}} \lesssim 40 \mathrm{GeV}$. Both of these predictions are in sharp contradiction with observations.

A quick inspection of the Coleman-Weinberg effective potential reveals the way to circumvent the above problem in weakly-coupled (perturbative) theories. One needs to ensure the overall dominance of the bosonic contributions to the effective potential over the fermionic ones, such that the experimental lower bound on the Higgs boson mass is satisfied while keeping the coupling constant in the perturbative domain. The simplest way to achieve this is by simply adding one (or more) scalar fields to the theory. ${ }^{2}$ The minimal model of this type involves a Higgs potential with just three parameters - which is only one more parameter than the SM case. We study this model in section II, where we show that it does provide a phenomenological consistent theoretical framework to realise the Higgs boson as a PGB of broken scale invariance.

Extended scalar sectors are a feature of many theories beyond the standard model. One

\footnotetext{
${ }^{2}$ Scale invariant models with additional scalars have been considered in Refs. [4] and [5] (see also the discussion of the scale-invariant case in Ref. [6]). The philosophy of those models was somewhat different though, with the additional scalar gaining a large VEV in both models which means that the electroweak Higgs could not be interpreted as the PGB of broken scale invariance. Also in Ref. [4] there was an additional $U(1)_{X}$ gauge boson. The models which we consider are simpler, with less parameters than these alternative models.
} 
such theory, with a particularly simple Higgs sector, is the mirror model [3]. In that theory, one essentially has two isomorphic sectors of particles, the standard particles and a 'mirror' sector. The mirror particles are governed by a Lagrangian of exactly the same form as the standard model, so that a discrete $Z_{2}$ mirror symmetry can be defined interchanging the ordinary and the mirror particles. If we make the theory scale invariant, by eliminating the $\mu^{2}$ mass parameter in the Higgs potential, then we can generate electroweak symmetry breaking radiatively, via the Coleman-Weinberg mechanism. The Higgs potential then has just two parameters (the same as in the SM) since the $Z_{2}$ mirror symmetry in the model fixes the quartic coupling constant in the mirror sector to be the same as the corresponding coupling constant in the ordinary particle sector. We show in section III that this classically scale invariant model is phenomenologically consistent, and also realises the Higgs boson as a PGB of spontaneously broken scale invariance.

\section{The next-to-minimal scale-invariant Standard Model}

Consider a minimal extension of the scale-invariant SM with a single extra real scalar field. The most general renormalisable potential is

$$
V_{0}(\phi, S)=\frac{\lambda_{1}}{2}\left(\phi^{\dagger} \phi\right)^{2}+\frac{\lambda_{2}}{8} S^{4}+\frac{\lambda_{3}}{2}\left(\phi^{\dagger} \phi\right) S^{2},
$$

where $\phi$ is the electroweak Higgs doublet and $S$ is the real singlet field. observe that the above potential (as well as total Lagrangian) has an accidental discrete $Z_{2}$ symmetry: $S \rightarrow-S$. We parameterise the fields (in the unitary gauge) as:

$$
\phi=\frac{r}{\sqrt{2}}\left(\begin{array}{c}
0 \\
\cos \omega
\end{array}\right), \quad S=r \sin \omega
$$

In this parameterisation, the potential (1) takes the form

$$
V_{0}(r, \omega)=r^{4}\left(\frac{\lambda_{1}}{8} \cos ^{4} \omega+\frac{\lambda_{2}}{8} \sin ^{4} \omega+\frac{\lambda_{3}}{4} \sin ^{2} \omega \cos ^{2} \omega\right) .
$$

Note that the radial component $r$ of the Higgs fields (2) factors out in the absence of the tree-level mass parameter. The renormalised quantum-corrected potential, in addition to (3), includes a sum of $\delta V_{k \text {-loop }}$ contributions generated at $k$-loop level $(k=1,2, \ldots)$. Each $k$-loop contribution is a $k^{\text {th }}$ order polynomial in $\log (\phi / \Lambda)$, where $\Lambda$ is some renormalisation scale. Perturbation theory is valid if the logarithms are not too large, so that $V_{0}>\delta V_{1-\text { loop }}>$ $\delta V_{2-\text { loop }}>\ldots$ is satisfied. In general, the minimisation of even a 1-loop corrected effective

potential involving multiple scalars cannot be done analytically, but one may resort to a 
numerical analysis. Here we instead follow the approximate analytic method suggested in [2] which is suitable in weakly coupled scale invariant theories.

Following [2], we first ignore perturbatively small radiative corrections and concentrate on the tree-level potential (3). Minimising the potential, assuming that $r \neq 0$ but is otherwise at this stage arbitrary, leads to two possible cases (we ignore a third case with unbroken electroweak symmetry because it is phenomenologically not viable). If $\lambda_{3}>0$ then

$$
\langle\sin \omega\rangle=0,\langle r\rangle=\sqrt{2}\langle\phi\rangle \equiv v \approx 246 \mathrm{GeV}, \quad<S>=0
$$

with

$$
\lambda_{1}(\Lambda)=0 .
$$

In this case only the electroweak Higgs develops a nonzero VEV. If $\lambda_{3}<0$ then

$$
\begin{aligned}
\left\langle\tan ^{2} \omega\right\rangle & =\epsilon \\
\sqrt{2}\langle\phi\rangle & =\langle r\rangle\left(\frac{1}{1+\epsilon}\right)^{1 / 2} \equiv v \approx 246 \mathrm{GeV},\langle S\rangle=v\langle\tan \omega\rangle
\end{aligned}
$$

with

$$
\lambda_{3}(\Lambda)+\sqrt{\lambda_{1}(\Lambda) \lambda_{2}(\Lambda)}=0
$$

where $\epsilon \equiv \sqrt{\frac{\lambda_{1}(\Lambda)}{\lambda_{2}(\Lambda)}}$. In this case both scalar fields develop VEVs and the discrete $Z_{2}$ symmetry is also broken spontaneously. ${ }^{3}$

Relations such as Eqs.(5) and (7) can be satisfied by an appropriate choice of the renormalizsation point $\mu=\Lambda[2]$, where the running coupling constants depend on $\mu$, and $\Lambda$ is the specific value where the required relations hold. In each case, the tree-level potential then has a flat direction along the vacuum solution, and the relation Eq.(5) [or Eq.(7)] removes a dimensionless parameter in favour of the renormalisation point $\Lambda$ which has the dimension of mass (dimensional transmutation). Because the tree-level potential vanishes along a specific direction, the 1-loop correction to it will dominate along that direction.

Next we calculate the tree-level masses by expanding the Higgs potential, Eq.(1), around the vacuum: $\phi=\langle\phi\rangle+\phi^{\prime}, S=\langle S\rangle+S^{\prime}$. In each case there are two physical scalars, but only one of these gains mass at tree-level, since there is a flat direction in the Higgs potential. Let us call $H$ the state that gets mass at tree-level, and $h$ the state which is massless at tree-level (the PGB of broken scale invariance). For the first case, where $\lambda_{3}>0 \Rightarrow\langle S\rangle=0$ [Eq.(4)], we find

$$
m_{H}^{2}=\frac{\lambda_{3} v^{2}}{2}, \quad H=S
$$

\footnotetext{
${ }^{3}$ This might cause a problem with cosmological domain walls unless $\langle S\rangle$ is sufficiently small. If it is very small but nonzero, then a network of domain walls would form with an equation of state $(p=w \rho)$ parameter $w=-2 / 3$ in the non-relativistic limit. This is somewhat interesting for dark energy reasons, though present indications are that a $w$ value closer to -1 is preferred by the data.
} 
The PGB in this case is $h=\phi_{0}^{\prime}$.

In the second case, where $\lambda_{3}<0$ and both $S$ and $\phi$ gain VEVs, we find

$$
m_{H}^{2}=\lambda_{1} v^{2}-\lambda_{3} v^{2}, \quad H=-\sin \omega \phi_{0}^{\prime}+\cos \omega S^{\prime} .
$$

In this case the PGB is $h=\cos \omega \phi_{0}^{\prime}+\sin \omega S^{\prime}$.

Let us now calculate the mass of the PGB boson for each pattern of symmetry breaking. The 1-loop correction to the tree-level potential (3) (along the radial direction) has the form $[2]$

$$
\delta V_{1-\text { loop }}=A r^{4}+B r^{4} \log \left(\frac{r^{2}}{\Lambda^{2}}\right)
$$

where

$$
A=\frac{1}{64 \pi^{2}\langle r\rangle^{4}}\left[3 \operatorname{Tr}\left(M_{V}^{4} \log \left(\frac{M_{V}^{2}}{\langle r\rangle^{2}}\right)\right)+\operatorname{Tr}\left(M_{S}^{4} \log \left(\frac{M_{S}^{2}}{\langle r\rangle^{2}}\right)\right)-4 \operatorname{Tr}\left(M_{F}^{4} \log \left(\frac{M_{F}^{2}}{\langle r\rangle^{2}}\right)\right)\right],
$$

and

$$
B=\frac{1}{64 \pi^{2}\langle r\rangle^{4}}\left[3 \operatorname{Tr} M_{V}^{4}+\operatorname{Tr} M_{S}^{4}-4 \operatorname{Tr} M_{F}^{4}\right] .
$$

The traces in the above equations go over all internal degrees of freedom, and $M_{V, S, F}$ are the tree-level masses respectively for vectors, scalars and fermions evaluated for the given VEV pattern.

The stationary condition $\left.\frac{\partial \delta V_{1-\text { loop }}}{\partial r}\right|_{r=\langle r\rangle}=0$ implies the relation

$$
\log \left(\frac{\langle r\rangle}{\Lambda}\right)=-\frac{1}{4}-\frac{A}{2 B}
$$

The PGB mass can be calculated directly from $\delta V_{1-\text { loop }}$. Using (13) one finds [2]:

$$
\begin{aligned}
m_{h}^{2} & =\left.\frac{\partial^{2} \delta V_{1-\text { loop }}}{\partial r^{2}}\right|_{r=\langle r\rangle}=8 B\langle r\rangle^{2} \\
& =\frac{1}{8 \pi^{2}\langle r\rangle^{2}}\left[3 \operatorname{Tr} M_{V}^{4}+\operatorname{Tr} M_{S}^{4}-4 \operatorname{Tr} M_{F}^{4}\right] .
\end{aligned}
$$

Applying this equation to determine the mass of the PGB, we find:

$$
\begin{aligned}
m_{h}^{2} & =\frac{1}{8 \pi^{2}\langle r\rangle^{2}}\left[6 m_{W}^{4}+3 m_{Z}^{4}+m_{H}^{4}-12 m_{t}^{4}\right] \\
& \approx \frac{m_{H}^{4} \cos ^{2} \omega}{8 \pi^{2} v^{2}},
\end{aligned}
$$

since we need $m_{H}$ to dominate over the other terms if the PGB mass is to be larger than the experimental lower limit of about $\sim 115 \mathrm{GeV}$. 
Precision electroweak tests put an upper bound on the Higgs boson mass. The current upper limit for the standard model Higgs is $m_{\text {higgs }}<M_{E W}$, with $M_{E W} \approx 186 \mathrm{GeV}$ at $95 \%$ C.L. [7]. Since the Higgs boson mass gives a radiative correction at l-loop level via a log term, we can find the corresponding limit in this model by the replacement $\lambda_{S M}^{2} \log m_{\text {higgs }}^{2} \rightarrow$ $\lambda_{S M}^{2} \cos ^{2} \omega \log m_{h}^{2}+\lambda_{S M}^{2} \sin ^{2} \omega \log m_{H}^{2}$. Thus, the limit on the scale invariant model from precision electroweak tests is

$$
\left(\frac{m_{h}}{m_{H}}\right)^{c_{\omega}^{2}} m_{H}<M_{E W},
$$

where $c_{\omega} \equiv \cos \omega$. Clearly, in the first symmetry breaking scenario, where $\cos \omega=1$, this bound only constrains the mass of the PGB $h$ to be less than $M_{E W}$ which can easily be satisfied (as is also the case in the SM). In the second case, where $\cos \omega$ is essentially a free parameter, the above bound gives a contraint on $\omega$ (given the relation, Eq.15). Using the current experimental bound, $M_{E W} \approx 186 \mathrm{GeV}$, we find that $\tan \omega<0.65$. That is to say, the PGB mainly "resides" in the electroweak doublet.

Note that the above analysis with one real scalar field can be simply extended to $N$ real scalar fields. Taking for simplicity an $O(N)$ symmetric potential, we simply need to replace $S^{2} \rightarrow \sum_{i=1}^{N} S_{i}^{2}$ in the potential, Eq.(1). In this case only the $\lambda_{3}>0$ region, where $\left\langle S_{i}\right\rangle=0$, is phenomenologically viable, since having $\left\langle S_{i}\right\rangle \neq 0$ would lead to massless Goldstone bosons from the spontaneous breaking of $O(N)$ symmetry. Also, if $\left\langle S_{i}\right\rangle=0$ we can also give $S$ gauge quantum numbers. For example, having $S$ complex and transforming as an $S U(3)_{c}$ colour triplet would be equivalent to having $N=6$ real scalar fields with an $O(6)$ symmetric potential. Having $N$ scalar fields, will give a factor $N$ in the right-hand side of Eq.(15) and thus reduce the mass of the heavy scalar (for a fixed $m_{h}$ ).

To find the domain of validity of perturbation theory we need to look at the renormalisation group equations [8]:

$$
\begin{aligned}
4 \pi^{2} \frac{d \lambda_{1}}{d t} & =3 \lambda_{1}^{2}+\frac{\lambda_{3}^{2}}{4} N \\
4 \pi^{2} \frac{d \lambda_{2}}{d t} & =\left[\frac{N+8}{4}\right] \lambda_{2}^{2}+\lambda_{3}^{2} \\
4 \pi^{2} \frac{d \lambda_{3}}{d t} & =\frac{3 \lambda_{1} \lambda_{3}}{2}+\frac{(N+2)}{4} \lambda_{2} \lambda_{3}+\frac{\lambda_{3}^{2}}{2}
\end{aligned}
$$

where $t=\log \mu$. Contributions from gauge and Yukawa coupling constants can be approximately neglected relative to the Higgs potential couplings constants.

These equations must be supplemented by the constraint Eq.(5) or (7) for the two symmetry breaking scenarios of interest. Due to the relatively large $m_{H}$ mass, the position of the Landau pole, $\mu_{L}$, is typically only a few orders of magnitude above the weak scale in both symmetry breaking scenarios. For example, for $m_{h}$ at the experimental limit of 115 GeV, taking $N=1(N=6)$ and the case where $\lambda_{3}>0$ so that $\langle S\rangle=0$, we find, numerically 
solving the equations, that $\mu_{L} \approx 20 \Lambda \approx 10^{4} \mathrm{GeV}\left(\mu_{L} \approx 4 \times 10^{2} \Lambda \approx 10^{5} \mathrm{GeV}\right)$. [Note that $\Lambda$ is determined from Eq.(13)].

\section{Electroweak Higgs as a PGB of broken scale invari- ance in mirror models}

In the previous section we showed that extending the Higgs sector in a scale invariant theory allows consistent models giving a naturally light and radiatively stable Higgs boson. Many theories beyond the SM actually require an extended Higgs sector. One theory with a particularly simple Higgs potential is the mirror matter model [3]. In the simplest version of that theory each type of ordinary particle (other than the graviton) has a distinct mirror partner. The ordinary and mirror particles form parallel sectors, each with gauge symmetry $G_{\mathrm{SM}}=S U(3) \otimes S U(2) \otimes U(1)$, so that the overall gauge group is $G_{\mathrm{SM}} \otimes G_{\mathrm{SM}}$. The interactions within each sector are governed by Lagrangians of exactly the same form, except that mirror weak interactions are right-handed. ${ }^{4}$ In other words, the full Lagrangian has the form

$$
\mathcal{L}=\mathcal{L}_{1}+\mathcal{L}_{2}+\mathcal{L}_{\text {mix }}
$$

where $\mathcal{L}_{1}$ is the usual Lagrangian of the SM, while $\mathcal{L}_{2}$ is the Lagrangian for the mirror SM. They are related by a parity symmetry $\mathcal{P}$, such that $\mathcal{P} \mathcal{L}_{1} \mathcal{P}^{-1}=\mathcal{L}_{2}$, which is imposed as an exact symmetry of the whole Lagrangian: $\mathcal{P} \mathcal{L} \mathcal{P}^{-1}=\mathcal{L}$. The third term $\mathcal{L}_{\text {mix }}$ contains only two parity invariant renormalisable interactions that couple the ordinary sector with the mirror one:

$$
\mathcal{L}_{\text {mix }}=\epsilon F_{\mu \nu}^{1} F^{2 \mu \nu}+2 \lambda\left(\phi_{1}^{\dagger} \phi_{1}\right)\left(\phi_{2}^{\dagger} \phi_{2}\right)
$$

where $F_{\mu \nu}^{1}$ and $F_{\mu \nu}^{2}$ are the $U(1)$ field strengths for the ordinary and mirror sectors and $\phi_{1}$ and $\phi_{2}$ are ordinary and mirror electroweak Higgs doublets, respectively. Thus we have only two extra parameters, $\epsilon$ and $\lambda$, in addition to those of the ordinary SM. The $U(1)$ kinetic mixing term will not be of interest to us here.

The most general tree-level Higgs potential of the scale invariant mirror model can be expressed as

$$
V_{0}\left(\phi_{i}\right)=\lambda\left(\phi_{1}^{\dagger} \phi_{1}+\phi_{2}^{\dagger} \phi_{2}\right)^{2}+\delta\left(\phi_{1}^{\dagger} \phi_{1} \phi_{1}^{\dagger} \phi_{1}+\phi_{2}^{\dagger} \phi_{2} \phi_{2}^{\dagger} \phi_{2}\right)
$$

This potential is bounded from below if $\lambda+\delta \geq 0$ and $\lambda+\frac{\delta}{2} \geq 0$. We take the Higgs field $\phi_{i}$ of each sector in unitary gauge and express them as

$$
\phi_{1}=\frac{r}{\sqrt{2}}\left(\begin{array}{c}
0 \\
\cos \omega
\end{array}\right), \phi_{2}=\frac{r}{\sqrt{2}}\left(\begin{array}{c}
0 \\
\sin \omega
\end{array}\right) .
$$

\footnotetext{
${ }^{4}$ There is a related model where the interchange symmetry is just an internal $Z_{2}$, unrelated to parity. In that version, the "shadow" weak interactions are left-handed.
} 
Then the potential (20) takes the form,

$$
V_{0}(r, \omega)=\frac{r^{4}}{4}\left[\lambda+\delta\left(\cos ^{4} \omega+\sin ^{4} \omega\right)\right] .
$$

As in the previous section, we can minimise the potential, assuming $r \neq 0$, which leads to two cases (depending on the sign of $\lambda$ ):

$$
\begin{aligned}
\text { Broken }-\mathrm{P} \text { case for } \lambda>0: & \sin \omega=0 \text { or } \cos \omega=0 \quad \text { requiring } \quad \lambda+\delta=0 \\
\text { Unbroken }-\mathrm{P} \text { case for } \lambda<0 \quad: \quad & \sin \omega=\cos \omega=\frac{1}{\sqrt{2}} \quad \text { requiring } \quad \lambda+\frac{\delta}{2}=0 .
\end{aligned}
$$

As before, the relations between $\lambda$ and $\delta$ will be satisfied by an appropriate choice of the renormalisation point $\mu=\Lambda$. For the broken-P case, we have either $\left\langle\phi_{1}\right\rangle$ or $\left\langle\phi_{2}\right\rangle$ being zero. Since we are identifying sector 1 as the ordinary sector, we shall concentrate on the $\sin \omega=0$ configuration.

Next we calculate tree-level mass-squared matrix at $\mu=\Lambda$ :

$$
\begin{aligned}
\text { Broken }-\mathrm{P} \text { Case } & : \quad M_{i j}^{2}=\lambda\langle r\rangle^{2}\left[\begin{array}{ll}
0 & 0 \\
0 & 1
\end{array}\right] \\
\text { Unbroken }-\mathrm{P} \text { case } & : \quad M_{i j}^{2}=\lambda\langle r\rangle^{2}\left[\begin{array}{cc}
-1 & 1 \\
1 & -1
\end{array}\right]
\end{aligned}
$$

The above mass matrices each have vanishing determinant, so one of the physical scalar fields, the PGB, is massless at tree level. As usual, the conformal anomaly means it obtains a relatively small mass from the 1-loop correction to $V_{0}$.

Also, it can be easily seen that the matrices in Eq.(24) have non-negative eigenvalues provided that $\lambda>0(\lambda<0)$ for the broken- (unbroken-) $\mathrm{P}$ cases. Thus, there are only two physically distinct minima of the potential (22):

$$
\langle r\rangle \equiv v \approx 246 \mathrm{GeV}, \quad\langle\cos \omega\rangle=1, \quad \text { if } \lambda>0
$$

and

$$
\langle r\rangle \equiv \sqrt{2} v=\sqrt{2} \cdot 246 \mathrm{GeV}, \quad\langle\cos \omega\rangle=\frac{1}{\sqrt{2}}, \quad \text { if } \lambda<0 .
$$

In case (25) the electroweak symmetry is broken in the SM sector, while the electroweak symmetry in the mirror sectors is intact ${ }^{5}$, and hence the $Z_{2}$ parity symmetry is spontaneously broken. In this model, the scalar sector consists of the standard Higgs boson, which is massless at tree level, and a complex mirror-doublet of bosons with mass squared: $m_{H}^{2}=$ $\lambda v^{2} \approx \lambda(246)^{2} \mathrm{GeV}^{2}$.

\footnotetext{
${ }^{5} S U(2) \otimes U(1)$ electroweak symmetry in the mirror sector is eventually broken through mirror $S U(3)$ quark condensation. For details, see Ref. [9].
} 
In case (26), the $S U(2) \times U(1)$ symmetry is broken in each sector so the $Z_{2}$ mirror symmetry remains exact. Consequently, the gauge bosons and fermions in each sector obtain the same masses as the corresponding particles in the SM. At tree level there is one massive scalar, with $m_{H}^{2}=-4 \lambda v^{2} \approx-4 \lambda(246) \mathrm{GeV}^{2}$, and one massless state. This massless state corresponds to the PGB of broken scale invariance. The mass eigenstates are parity eigenstates, maximal superpositions of the ordinary and mirror physical Higgs bosons.

Let us now calculate the mass of the PGB boson for each pattern of symmetry breaking. As briefly reviewed in section II, the mass of the PGB is, in general, given by Eq.(14). Applying this to the spontaneously broken mirror symmetry case (25) we obtain,

$$
\begin{aligned}
m_{h}^{2} & \simeq \frac{1}{8 \pi^{2} v^{2}}\left[6 m_{W}^{4}+3 m_{Z}^{4}+4 m_{H}^{4}-12 m_{t}^{4}\right] \\
& \approx \frac{m_{H}^{4}}{2 \pi^{2} v^{2}} \text { for } m_{h} \gtrsim 115 \mathrm{GeV},
\end{aligned}
$$

while for the unbroken mirror symmetry case (26) we find:

$$
\begin{aligned}
m_{h}^{2} & \simeq \frac{1}{16 \pi^{2} v^{2}}\left[12 m_{W}^{4}+6 m_{Z}^{4}+m_{H}^{4}-24 m_{t}^{4}\right] \\
& \approx \frac{m_{H}^{4}}{16 \pi^{2} v^{2}} \text { for } m_{h} \gtrsim 115 \mathrm{GeV} .
\end{aligned}
$$

Thus, we effectively have a mass relation between the light and heavy Higgs bosons in the models. Numerically, we obtain the approximate relations:

$$
\begin{array}{ll}
m_{H} \approx\left(\frac{m_{h}}{115 \mathrm{GeV}}\right)^{1 / 2} 360 \mathrm{GeV} & \text { Broken case (Eq.25) } \\
m_{H} \approx\left(\frac{m_{h}}{115 \mathrm{GeV}}\right)^{1 / 2} 600 \mathrm{GeV} & \text { Unbroken case (Eq.26). }
\end{array}
$$

Phenomenologically, the broken mirror symmetry case, Eq.(25), mimics the SM. The light Higgs, $h$, couples in exactly the same way as does the SM Higgs field, while the heavier states, $H$, couple to the mirror sector. In this case, the experimental lower limit on $m_{h}$ is the same as the SM limit of approximately $115 \mathrm{GeV}$. Also, the upper bound on $m_{h}$ inferred from precision electroweak measurements is $m_{h}<M_{E W} \approx 186$ [7]. There is no difficulty in satisfying these constraints.

In the case of the unbroken mirror symmetry, Eq.(26), the light Higgs field $h$, couples to both of the sectors, with coupling strength $1 / \sqrt{2}$ compared with the SM Higgs. Thus the limit from precision electroweak measurements in this model is given by Eq.(16) with $c_{\omega}^{2}=1 / 2$. However, for $M_{E W} \approx 186 \mathrm{GeV}$, this bound is inconsistent with the relation, Eq.(28). We conclude that the Coleman-Weinberg mechanism is consistent with existing 
phenomenological bounds only for the broken-P situation ${ }^{6}$. Below, we therefore discuss the broken-P case only.

The experimental lower bound on the mass of the SM Higgs boson, $m_{h}>115 \mathrm{GeV}$, can be translated into a lower bound on the coupling $\lambda_{2}(\Lambda) \equiv 2 \lambda(\Lambda)$ :

$$
\lambda_{2}(\Lambda)>4.2\left(\frac{115 \mathrm{GeV}}{m_{h}}\right),
$$

for the broken P case of Eq.(25). To find the domain of validity of perturbation theory we look, as before, at renormalisation group equations:

$$
\begin{gathered}
4 \pi^{2} \frac{d \lambda_{1}}{d t}=3 \lambda_{1}^{2}+\lambda_{2}^{2}, \\
4 \pi^{2} \frac{d \lambda_{2}}{d t}=3 \lambda_{1} \lambda_{2}+\frac{1}{2} \lambda_{2}^{2},
\end{gathered}
$$

where $\frac{1}{2} \lambda_{1}=\lambda+\delta$. As $\lambda_{2}(\Lambda)$ is significantly larger than any other coupling in the SM we can approximately neglect top-quark and gauge boson contributions. These equations must be supplemented by the constraint equation (23), which reads

$$
\lambda_{1}(\Lambda)=0,
$$

for the case (25). Numerically solving these equations, we find that the position, $\mu_{L}$, of the Landau pole is $\mu_{L} \approx 3 \times 10^{2} \Lambda \approx 10^{5} \mathrm{GeV}$ (for $m_{h}$ at the experimental limit of $115 \mathrm{GeV}$ ). The perturbative domain thus extends confortably above the electroweak scale.

\section{Conclusion}

We have examined the idea that the standard model Higgs boson might be the pseudoGoldstone boson of broken scale invariance. The simplest version of this idea is the original Coleman-Weinberg model. The problem there is that the mass of the Higgs is too small, and the spontaneous symmetry breaking can only occur if the top quark is also very light. However, if there is an extended Higgs sector, then the additional scalar degrees of freedom can compensate for the heavy top quark, and give a Higgs mass in excess of the experimental lower limit, currently around $115 \mathrm{GeV}$.

Specifically, we have considered two phenomenologically consistent models. The first involves the addition of one (or more) real scalar fields. The additional bosonic degrees of freedom can lead to phenomenologically successful electroweak symmetry breaking. An

\footnotetext{
${ }^{6}$ Of course this conclusion is only for the minimal mirror model with two sectors. In this context it will be interesting to study the case of generalized mirror models with $\mathrm{N}$-sectors [10]. However, we will leave this study for the future.
} 
even more constrained symmetry breaking sector arises in the mirror model, which has a discrete symmetry interchanging the standard model Higgs with a mirror partner. The discrete symmetry eliminates one parameter in the Higgs potential, and leads to a consistent electroweak symmetry breaking with the same number of parameters as in the standard model case.

The proposed scale-invariant models (and their generalizations) can be testable in upcoming LHC experiments in the case of non-zero $\cos \omega$. The light PGB Higgs interacts with Standard Model particles with couplings reduced by factor $\cos \omega$ relative to the corresponding couplings of the Standard Model Higgs boson. In addition heavier Higgs-like boson with a mass correlated with the mass of PGB Higgs (see Eq.(15) can be observed. The couplings of this heavy boson with Standard model particles is suppressed by $\sin \omega$ and its total decay width will be dominated by the decay width into two PGB Higgses. In the case of $\cos \omega=0$, the difference between the PGB Higgs and the Standard Model Higgs is rooted in the scalar potential, and, most probably, LHC will not be capable to distinguish among them. Future linear collider (e.g. the proposed ILC) should be able to study this case.

\section{Acknowledgements}

This work was supported by the Australian Research Council.

\section{References}

[1] S. R. Coleman and E. Weinberg, Phys. Rev. D 7 (1973) 1888.

[2] E. Gildener and S. Weinberg, Phys. Rev. D 13 (1976) 3333.

[3] R. Foot, H. Lew and R. R. Volkas, Phys. Lett. B 272 (1991) 67;

The concept of mirror matter was discussed prior to the advent of the standard model in T. D. Lee and C. N. Yang, Phys. Rev. 104 (1956) 256; I. Kobzarev, L. Okun and I. Pomeranchuk, Sov. J. Nucl. Phys. 3 (1996) 837; M. Pavsic, Int. J. Theor. Phys. 9 (1974) 229.

[4] R. Hempfling, Phys. Lett. B 379 (1996) 153 [arXiv:hep-ph/9604278];

W. F. Chang, J. N. Ng and J. M. S. Wu, arXiv:hep-ph/0701254.

[5] K. A. Meissner and H. Nicolai, arXiv:hep-th/0612165.

[6] J. R. Espinosa and M. Quiros, arXiv:hep-ph/0701145.

[7] W. M. Yao et al. [Particle Data Group], J. Phys. G 33 (2006) 1. 
[8] T. P. Cheng, E. Eichten and L. F. Li, Phys. Rev. D 9 (1974) 2259.

[9] R. Foot and H. Lew, arXiv:hep-ph/9411390.

R. Foot, H. Lew and R. R. Volkas, JHEP 0007 (2000) 032 [arXiv:hep-ph/0006027].

[10] R. Foot, Phys. Lett. B 632 (2006) 467 [arXiv:hep-ph/0507294];

R. Foot and R. R. Volkas, Phys. Lett. B 645 (2007) 75 [arXiv:hep-ph/0610013]. 